\title{
Genetic diversity analysis of two commercial breeds of pigs using genomic and pedigree data
}

Ricardo Zanella1,6, Jane O. Peixoto ${ }^{1}$, Fernando F. Cardoso 2,4, Leandro L. Cardoso², Patrícia Biegelmeyer ${ }^{4}$, Maurício E. Cantão ${ }^{1}$, Antonio Otaviano ${ }^{3}$, Marcelo S. Freitas ${ }^{3}$, Alexandre R. Caetano ${ }^{5,7}$ and Mônica C. Ledur ${ }^{1,8^{*}}$

\begin{abstract}
Background: Genetic improvement in livestock populations can be achieved without significantly affecting genetic diversity if mating systems and selection decisions take genetic relationships among individuals into consideration. The objective of this study was to examine the genetic diversity of two commercial breeds of pigs. Genotypes from 1168 Landrace (LA) and 1094 Large White (LW) animals from a commercial breeding program in Brazil were obtained using the Illumina PorcineSNP60 Beadchip. Inbreeding estimates based on pedigree $\left(F_{x}\right)$ and genomic information using runs of homozygosity $\left(F_{\mathrm{ROH}}\right)$ and the single nucleotide polymorphisms (SNP) by SNP inbreeding coefficient $\left(F_{S N P}\right)$ were obtained. Linkage disequilibrium $(L D)$, correlation of linkage phase $(r)$ and effective population size $\left(N_{e}\right)$ were also estimated.
\end{abstract}

Results: Estimates of inbreeding obtained with pedigree information were lower than those obtained with genomic data in both breeds. We observed that the extent of LD was slightly larger at shorter distances between SNPs in the LW population than in the LA population, which indicates that the LW population was derived from a smaller $N_{e}$. Estimates of $N_{e}$ based on genomic data were equal to 53 and 40 for the current populations of LA and LW, respectively. The correlation of linkage phase between the two breeds was equal to 0.77 at distances up to $50 \mathrm{~kb}$, which suggests that genome-wide association and selection should be performed within breed. Although selection intensities have been stronger in the LA breed than in the LW breed, levels of genomic and pedigree inbreeding were lower for the LA than for the LW breed.

Conclusions: The use of genomic data to evaluate population diversity in livestock animals can provide new and more precise insights about the effects of intense selection for production traits. Resulting information and knowledge can be used to effectively increase response to selection by appropriately managing the rate of inbreeding, minimizing negative effects of inbreeding depression and therefore maintaining desirable levels of genetic diversity.

\section{Background}

Pork is a low-cost source of high-quality animal protein and is produced for human consumption worldwide. Pork production is very important for the economy of many countries from the European Union, China, USA and Brazil, which ranks as the fourth largest pork producer in the world, with 3.39 million metric tons of

\footnotetext{
*Correspondence: monica.ledur@embrapa.br

${ }^{1}$ Embrapa Swine and Poultry National Research Center, Animal Breeding

and Genetics, Concordia, SC, Brazil

Full list of author information is available at the end of the article
}

meat produced per year [1]. Two breeds are mainly used as maternal lines in Brazilian swine breeding programs: Landrace (LA) and Large White (LW), which represent 20.2 and $18.9 \%$ of the overall germplasm used for pork production, respectively [1]. These two breeds alone were responsible for approximately $40 \%$ of all animals registered in the National Swine Producers Association until 2013 [1]. However, little is known about the current levels of genetic diversity within these two breeds in the Brazilian swine herd. 
Intense animal selection and different mating strategies have been used to improve production traits within the livestock sector [2]. Although higher selection intensities can lead to faster genetic progress [3], undesirable increases in inbreeding levels may result as a consequence. In addition, overuse and misuse of assisted reproductive technologies such as artificial insemination may exacerbate these effects [4], since the excessive use of specific sires to improve specific traits may lead to undesirable losses of genetic diversity. High levels of inbreeding lead to the accumulation of high levels of homozygosity in animals within a herd, which in turn is likely to have detrimental consequences on traits that are related to reproduction, body conformation, growth and immune response [5].

Recent technological advances in methods to generate genome-wide sequencing and genotyping data have significantly improved the well-established processes for pedigree testing and confirmation of paternity assignments [6]. High-density genomic data have also been successfully used to identify quantitative trait loci (QTL) that affect health, behavioral and production traits in several species, including pigs [7-10]. More recently, highdensity single nucleotide polymorphism (SNP) panels have also been used to estimate genetic diversity parameters for breeds or lines $[11,12]$.

The use of genomic information for pedigree correction and/or breeding and selection [13] can greatly improve accuracy of estimated breeding values and reduce generation intervals via the genomic evaluation of young animals before phenotypes can be measured. Therefore, significant increases in the rate of genetic gain are expected and have been reported in cattle [14]. However, at the same time, inbreeding can also increase at a higher rate if it is not properly considered in the selection and mating strategies $[15,16]$.

Inbreeding levels are conventionally estimated with pedigree information and depend strongly on the accuracy and amount of pedigree data $[15,17]$. Better accuracies can be obtained when inbreeding estimates are derived from genome-wide SNP data [18]. However, since alleles at a locus that are identical by descent (IBD) versus identical by state (IBS) cannot be distinguished, these methods can result in overestimated inbreeding levels compared to pedigree-based estimates [19]. An alternate approach to control these issues is to use estimates that are obtained from observed runs of homozygosity (ROH). Runs of homozygosity are defined as contiguous stretches of homozygous genotypes that are present in an animal due to both parents transmitting identical haplotypes to their offspring. $\mathrm{ROH}$ provide a more accurate prediction of alleles at a locus that are IBD, and have been widely used in studies on human populations to accurately estimate levels of autozygosity among individuals [20].

High levels of homozygosity are generally associated with the segregation of long stretches of homozygous regions across the genome, which in turn increases the chance that deleterious alleles are expressed in populations. In addition, increased levels of homozygosity are associated with reduced effective population size $\left(N_{e}\right)$, which is a measure of within-breed diversity that describes the inbreeding rate by generation and the loss of genetic variation [21]. It has been shown that the use of high-density SNP panels improves the accuracy of population parameter estimates, such as $N_{e}$, inbreeding across generations, linkage disequilibrium (LD) between loci within a population, and correlation of linkage phase, which is a measure of the degree of agreement of linkage phase for pairs of SNPs between two populations [17]. These parameters are useful measures of similarity within and across breeds [17]. Therefore, the objectives of this study were to investigate the within- and between-population diversity of two maternal purebred lines of distinct porcine breeds (LA and LW) in a commercial breeding program in Brazil, using pedigree and genomic data. In addition, conserved regions based on shared $\mathrm{ROH}$ within and between lines were further characterized and explored.

\section{Methods}

This study was conducted at Embrapa Swine and Poultry National Research Center and was approved by the Institutional Ethics Committee on Animal Utilization for all experimental protocols used.

\section{Animal populations}

All samples used in this study were obtained from two maternal lines of pigs from a commercial breeding nucleus (BRF/SA, Curitiba, PR, Brazil). A total of 1178 LA and 1200 LW DNA samples were genotyped. Animals were chosen according to their availability in the herd, while maximizing the number of breeders chosen. LA animals were born between 2006 and 2011 and LW animals between 2007 and 2011. For all the animals, complete pedigree records were available with an average depth of 6.4 and 5.6 generations for LA and LW, respectively, and information on a total of 84,611 LA and 50,348 LW animals in the pedigree.

\section{Sample collection and genotyping}

DNA extraction was performed on $200 \mathrm{mg}$ of frozen tissue from 2378 animals using the PureLink ${ }^{\circledR}$ Genomic DNA Mini Kit (Invitrogen, San Diego, CA), according to the manufacturer's instructions. Quantity and quality of DNA were measured with a NanoDrop ND-2000 
spectrophotometer (NanoDrop Technologies Inc., Wilmington, DE). The $260 / 280 \mathrm{~nm}$ readings for all samples ranged from 1.8 to 2.0. Samples were diluted to a final concentration of $500 \mathrm{ng}$ and genotyped by a commercial lab (GeneSeek, Lincoln, Nebraska, USA) using the Illumina PorcineSNP60 V2 BeadChip.

\section{Animal and SNP quality control}

Prior to analysis of the genotyping data, 116 animals were excluded from the dataset based on the following criteria and using PLINK [22]: call rate less than 0.90, a level of heterozygosity higher than 3 standard deviations from the mean, and duplicated samples (match level $>99 \%$ ). Pedigree errors based on IBD levels (sire or dam to offspring and full-sibs IBD $\sim 0.5$, half-sibs IBD $\sim 0.25$ and first cousins IBD $\sim 0.125$ ) and sex mis-assignments based on $\mathrm{X}$ chromosome inbreeding estimates (F) using standard values of $\mathrm{F}<0.2$ and $>0.8$ for females and males, respectively, were also verified using PLINK [22].

For the analysis of genomic inbreeding, SNPs with an unknown position based on the Pig60K_SNP_pos_build 10.2 (see http://www.animalgenome.org/repository/), SNPs with a call rate higher than 0.90, and SNPs located on sex chromosomes were removed. A total of 45,766 SNPs were used to estimate genomic inbreeding in the LA and LW pig breeds.

Additional data pruning was performed with $R$ snpStats (v 1.14.0) to prepare data for analyses of LD, correlation of linkage phase, and $N_{e}$ [23]. The following quality control $(\mathrm{QC})$ criteria were used to remove SNPs that had a call rate lower than 0.98 , a minor allele frequency (MAF) lower than 0.03 and that deviated significantly from Hardy-Weinberg equilibrium $\left(\mathrm{p}<10^{-6}\right)$. The final dataset contained 41,041 SNPs for LA and 36,452 SNPs for LW, and a total of 2262 samples, i.e. 1168 for LA (91 males and 1077 females) and 1094 for LW (114 males and 980 females). Sporadically missing genotypes were imputed during the phasing procedure using FImpute software [24].

Quality control was performed on both breeds for genomic inbreeding estimates, while for estimates of $N_{e}$, LD and correlation of linkage phase, it was performed within breed to avoid SNPs being penalized by the HWE criterion, since some SNPs can be fixed within one breed only.

\section{Linkage disequilibrium, correlation of linkage phase and effective population size}

Pairwise linkage disequilibrium $\left(r^{2}\right)$ estimates [25] were calculated using ld_estimate $\mathrm{R}$ scripts [26]. For each breed, LD values between all pairs of SNPs within each chromosome were grouped according to their pairwise physical distance in classes of $100 \mathrm{~kb}$, starting from 0 to
$10 \mathrm{Mb}$. Summarized $r^{2}$ values at different distances were obtained by calculating the mean across all chromosomes. In addition, $r^{2}$ estimates were also calculated using only one in every 10 SNPs to mimic a lower density SNP panel.

The correlation of linkage phase $(r)$ for SNP pairs between the two breeds was calculated following Badke et al. [26], using the same grouping strategy as applied for $r^{2}$. The mean values of $r$ according to distances between SNPs were calculated using the SNPs that were shared by both breeds.

The relationship between $r^{2}$ and $N_{e}$ was calculated following [27]:

$$
E\left(r^{2}\right)=1 /\left(4 c N_{e}+1\right),
$$

where $c$ is the genetic distance between two SNPs expressed in Morgan. Based on this equation, $N_{e}$ for $t$ generations in the past $\left(N_{e t}\right)$ can be estimated using the relationship between $t$ and $c(t=1 / 2 c)$ [28] and solving $N_{e}$ as:

$$
N_{e t}=\left(1-r^{2}\right) /\left(4 c r^{2}\right), \quad \text { for } 0.0<r^{2}<1.0 .
$$

First, physical distances between SNPs within each chromosome were converted to genetic distances considering $1 \mathrm{cM} \sim 1 \mathrm{Mb}$ [17]. Because generations were assumed to be discrete and distances between SNPs are continuous, to calculate $N_{e t}$, estimates of $r^{2}$ for a range of values of $c$ were used which, when applied in $t=1 / 2 c$, rounded to the target generation. For example, $r^{2}$ of all SNP pairs with distances between $0.333(t=1.5)$ and $1 \mathrm{M}$ $(t=0.5)$ were selected and averaged across all chromosomes to calculate $N_{e}$ at $t=1$. Due to the inverse relationship between $t$ and $c$, as $t$ increased, wider intervals around $t$ were used to define the corresponding ranges of $c$ to ensure that sufficient numbers of SNP pairs were used to reliably estimate $N_{e t}$ for each $t$ [27]. Values of $N_{e t}$ were obtained with increments of one generation for $t=1,2, \ldots, 10$, of five generations for $t=15,20 \ldots, 100$, and of 50 generations for $t=150,200, \ldots, 1000$ [29].

\section{Inbreeding estimates based on pedigree and genotypic data}

Pedigree-based inbreeding $\left(F_{\mathrm{x}}\right)$ was estimated according to Wright's coefficient [30] with the $\mathrm{R}$ pedigree package [31] for each population as a whole. Runs of homozygosity were calculated with PLINK [22], using the following parameters: a minimum ROH of 50 SNPs with a minimum length of $1000 \mathrm{~kb}$, and one heterozygous SNP and one missing SNP genotype were allowed within a sliding window of 50 SNPs $[4,11]$. Identified $\mathrm{ROH}$ were then used to estimate individual genomic inbreeding coefficients $\left(F_{\mathrm{ROH}}\right)$ following [4]: 


$$
F_{\mathrm{ROH}}=\frac{\sum_{k} \operatorname{Length}\left(\mathrm{ROH}_{k}\right)}{\mathrm{L}},
$$

where $k$ is the number of $\mathrm{ROH}$ identified for the individual, multiplied by the average length of its $\mathrm{ROH}$ segments, and $\mathrm{L}$ is the total swine genome length (2,808,525 kb, Sscrofa10.2, Aug 2011). Genomic SNP-bySNP inbreeding coefficients $\left(F_{\mathrm{SNP}}\right)$ were obtained based on the proportion of homozygous genotypes for each individual, which measures the observed percentage of homozygosity per animal. The three methods of estimating inbreeding were then compared within-breed using Pearson correlations.

\section{Identification of conserved regions and gene content in shared homozygous regions}

A comparison of the percentage and number of shared $\mathrm{ROH}$ among individuals was performed with a Pearl homemade script to identify conserved regions within and between breeds. For the identification of shared $\mathrm{ROH}$ within breeds, shared identical segments observed in different animals of the same breed were considered. To define shared $\mathrm{ROH}$ between breeds, an identical segment with the same start and end points found between animals of the different breeds was used. Further analyses were carried out using the UCSC genome browser [32], to search for positional candidate genes located on the identified shared $\mathrm{ROH}$.

\section{Results}

\section{Linkage disequilibrium, correlation of linkage phase} and effective population size

Results for LD between adjacent markers $\left(r^{2}\right)$ and persistence of linkage phase $(r)$ are in Table 1. Average $r^{2}$ for adjacent SNPs was slightly higher for the LW $\left(r^{2}=0.50\right)$ than for the LA breed $\left(r^{2}=0.46\right)$. Figure 1 shows the average $r^{2}$ by distance between SNPs in classes of $100 \mathrm{~kb}$,

Table 1 Inbreeding coefficient estimates, linkage disequilibrium and correlations of linkage phase for Landrace and Large White populations

\begin{tabular}{lll}
\hline & Landrace & Large White \\
\hline$F_{\mathrm{x}}$ & $0.014(0.0003)$ & $0.021(0.0003)$ \\
$F_{\mathrm{ROH}}$ & $0.094(0.0006)$ & $0.106(0.0007)$ \\
$F_{\mathrm{SNP}}$ & $0.668(0.0005)$ & $0.667(0.0512)$ \\
$r^{2}$ & $0.459(0.002)$ & $0.497(0.002)$ \\
$r$ & 0.770 & \\
\hline
\end{tabular}

Numbers presented are the average and its standard error; $F_{\mathrm{x}}=$ pedigreebased inbreeding coefficient; $F_{\mathrm{ROH}}=$ genomic inbreeding based on runs of homozygosity $(\mathrm{ROH}) ; F_{\mathrm{SNP}}=$ genomic inbreeding based on the proportion of SNPs that are homozygous (SNP-by-SNP); $r^{2}=$ linkage disequilibrium among adjacent SNPs; $r=$ correlations of linkage phase

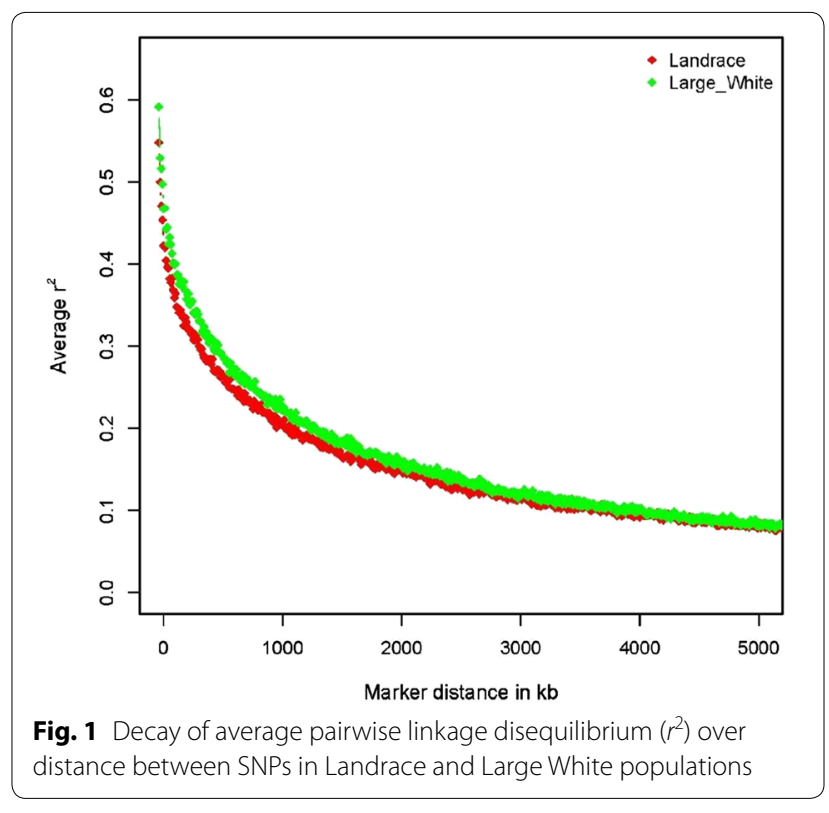

which provides an overview of the decline of $r^{2}$ over distance in each breed. At shorter distances, the average $r^{2}$ was higher in LW than in LA. Breed differences decreased as distance between markers increased and approached background LD levels at $5 \mathrm{Mb}$ distances in both breeds, with average $r^{2} \approx 0.08$. Average $r^{2}$ values for neighboring SNPs calculated by using one in every 10 available SNPs were equal to 0.27 for LA and 0.30 for LW.

The average distance between consecutive SNPs across all chromosomes was equal to 65.7 and $73.9 \mathrm{~kb}$ for LW and LA, respectively. For LA, $61 \%$ of adjacent informative SNP pairs had $r^{2}$ values higher than 0.2 and $53 \%$ had $r^{2}$ values higher than 0.3. For LW, informative SNPs that showed a level of LD higher than 0.2 and 0.3 represented 64 and $56 \%$ of the adjacent SNP pairs analyzed, respectively. Correlations of linkage phase between the two breeds were moderately high $(r=0.77)$ between SNPs at distances up to $50 \mathrm{~kb}$ (Fig. 2).

Estimates of $N_{e}$ obtained for the past 1000 generations for LA and LW are in Fig. 3. Estimated $N_{e}$ at 1000 generations back was equal to 572 for LW and 740 for LA. In the last five generations, $N_{e}$ ranged from 50 to 53 for LA and from 40 to 48 for LW.

\section{Inbreeding coefficient estimates based on pedigree and genomic data}

Inbreeding coefficients estimated based on pedigree and genomic data for both breeds are in Table 1. Inbreeding estimates $\left(F_{\mathrm{x}}\right)$ obtained by using all pedigree data available for both breeds ranged from 0 to 0.139 with an average of 0.014 for LA, and from 0 to 0.062 with an average of 0.021 for LW (Fig. 4). Although higher inbreeding levels were observed 


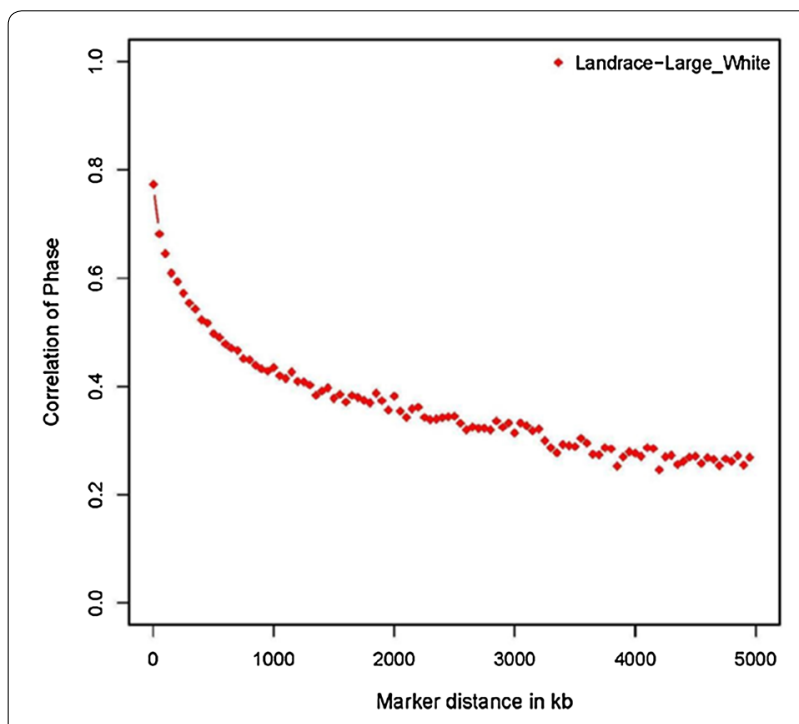

Fig. 2 Correlation of linkage phase between breeds for SNP pairs grouped by distance in $100 \mathrm{~kb}$ intervals across the genome

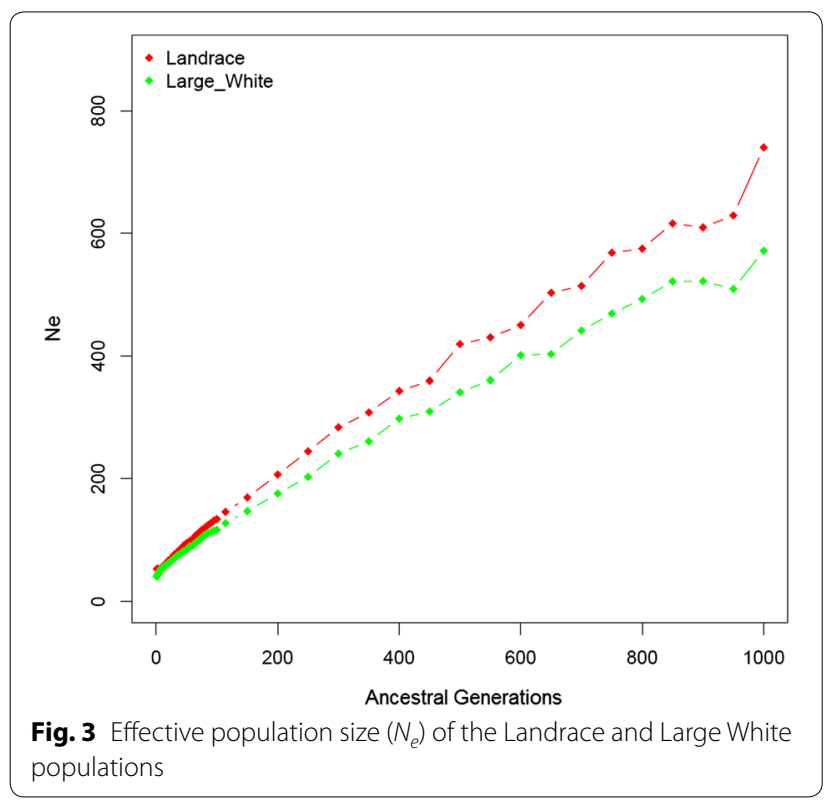

for some LA animals compared to the LW animals, the average $F_{\mathrm{x}}$ was higher for the LW than the LA breed.

The average number of observed $\mathrm{ROH}$ per genome was equal to 52.7 (ranging from 10 to 84 ) for LA, and 61.4 (ranging from 7 to 87) for LW. The size of the ROH ranged from 28.8 to $513.0 \mathrm{Mb}$ (mean $=252.9 \mathrm{Mb}$ ) for $\mathrm{LA}$ and from 23.8 to $498.5 \mathrm{Mb}($ mean $=280.1 \mathrm{Mb})$ for LW (Fig. 5). Both the mean number and size of $\mathrm{ROH}$ differed significantly between LA and LW $\left(\mathrm{p}<2.2^{\mathrm{e}-16}\right)$. The correlation between number and size of $\mathrm{ROH}$ within breed was 0.64 for LA and 0.78 for LW. Average genomic inbreeding based on observed $\mathrm{ROH}\left(F_{\mathrm{ROH}}\right)$ was equal to 0.09 (ranging from 0.0001 to 0.180 ) for LA and 0.10 (ranging from 0.008 to 0.177 ) for LW. Estimated $F_{\mathrm{SNP}}$ inbreeding coefficients ranged from 0.612 to 0.727 for LA (mean $=0.668$ ) and from 0.611 to 0.717 $($ mean $=0.667)$ for LW.

Correlations between estimated levels of $F_{\mathrm{ROH}}$ and $F_{\mathrm{SNP}}$ were moderately high for both LA $\left(r^{2}=0.82\right)$ and LW $\left(r^{2}=0.71\right)$. Conversely, correlations between $F_{\mathrm{x}}$ and $F_{\mathrm{ROH}}$ and $F_{\mathrm{x}}$ and $F_{\mathrm{SNP}}$ were low for both LA $\left(r^{2}=0.24\right.$ and 0.21 , respectively) and LW ( $r^{2}=0.015$ and 0.140 , respectively).

\section{$\mathrm{ROH}$, conserved regions, gene content and shared}

\section{homozygosity}

A region on SSC1 (SSC for Sus scrofa chromosome) that spanned 2.42 Mb (between 190,770,055 and $193,195,907 \mathrm{bp}$ ) was shared by $61.5 \%$ of the LA individuals. A similar region was also shared among $56 \%$ of the LW individuals (between 189,577,254 and 192,117,017 bp). This region did not contain any known annotated genes in the pig genome.

SSC14 contained the largest number of $\mathrm{ROH}$ for the LA breed. The region between 99,956,031 and $101,787,411$ bp on SSC14 was shared among 629 LA individuals $(53.9 \%)$ and harbors two genes: CXCL12 (chemokine C-X-C motif ligand 12) and TFAM (transcription factor A mitochondrial precursor). CXCL12 was shown to be associated with immunological traits in the LA breed, especially with disease resistance, and may therefore be important for survival [33]. TFAM plays an important role in porcine gametogenesis and embryo preimplantation and development and thus may have broad implications in cell physiology and evolutionary biology [34]. A region on SSC4 (between 96,423,033 and $98,660,311 \mathrm{bp}$ ) was shared among $62 \%$ of LW individuals and harbors 13 known genes with different functions.

A total of 1333 homozygous genomic regions were shared between the LA and LW breeds (Table 2), which suggests that they harbor important genes that have been under selection during domestication and contemporary breeding for production performance.

\section{Discussion}

The genetic diversity within and between two maternal breeds of pigs (LA-Landrace and LW-Large White) used in a commercial breeding nucleus was analyzed using pedigree and genomic information. Three methods were computed to estimate inbreeding coefficients: Wright's pedigree-based inbreeding coefficient $\left(F_{\mathrm{x}}\right)$, $\mathrm{ROH}\left(F_{\mathrm{ROH}}\right)$ and the SNP-by-SNP coefficient $\left(F_{\mathrm{SNP}}\right)$. In addition, estimates of LD, $N_{e}$ and correlation of linkage phase were calculated for both breeds, which are important parameters to appropriately manage the population's 


\section{Landrace}

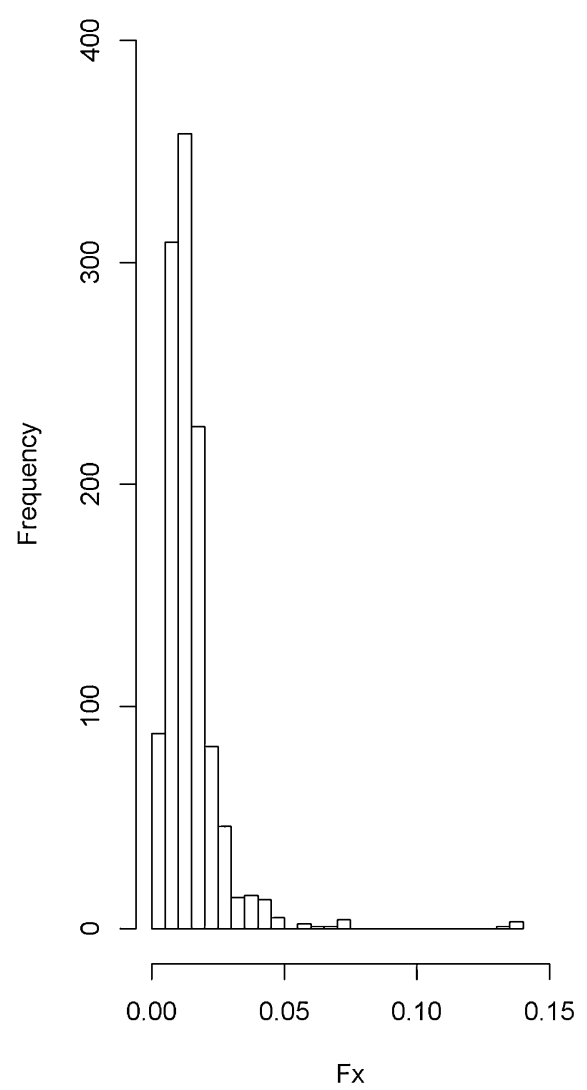

\section{Large White}

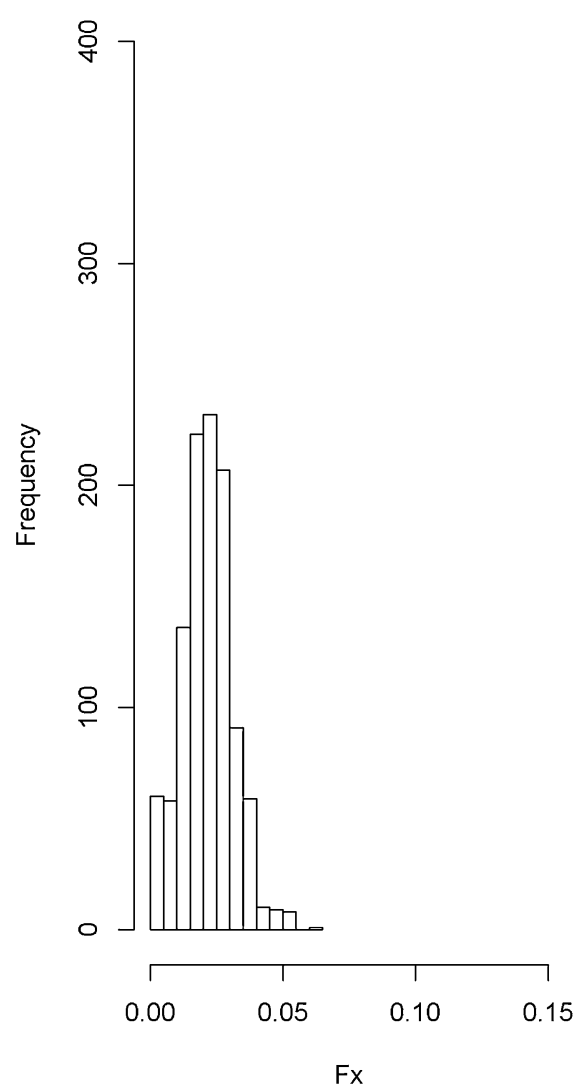

Fig. 4 Inbreeding estimates based on pedigree information $\left(F_{x}\right)$

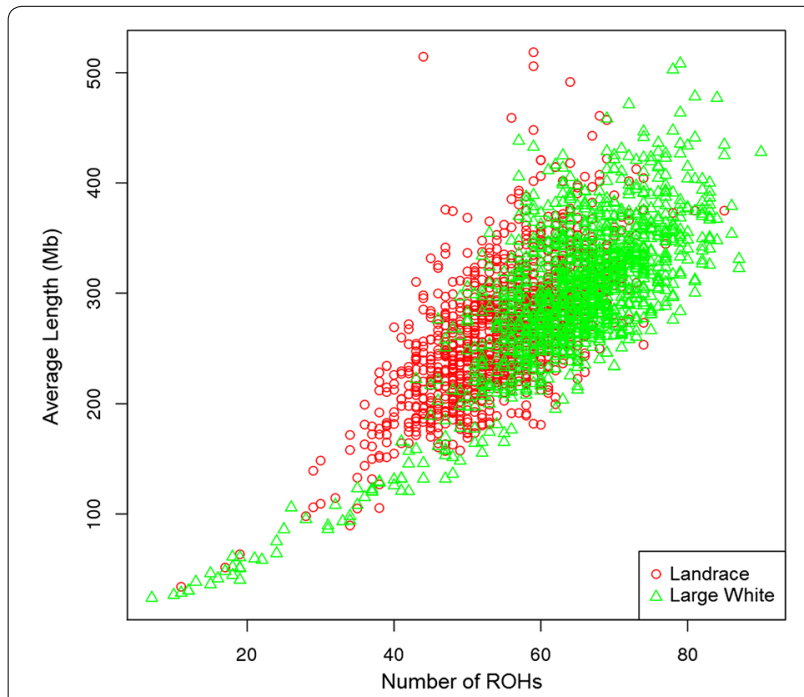

Fig. 5 Relationship between the number of $\mathrm{ROH}$ and the average length of each $\mathrm{ROH}$ for the Landrace and Large White populations. Each point represents the number and average length of $\mathrm{ROH}$ of one animal. Red circles represent Landrace and green triangles represent Large White animals genetic diversity, which is a condition to effectively increase long-term response to selection.

Our results revealed a larger $N_{e}$ and lower levels of homozygosity for LA than for LW. Moreover, the larger size and larger number of ROH found for the LW breed suggest that recent consanguinity events occurred in this breed, which is congruent with the observed estimates of $N_{e}$, inbreeding and LD. However, since quality control for the estimation of $N_{e}$ and LD was carried out within breed, the number of and distance between the SNPs used were different for LA and LW. Therefore, these results should be taken with caution.

Previous evaluations of LD and $N_{e}$ in Finnish Landrace and Yorkshire pigs using the Illumina PorcineSNP60 Beadchip generated average estimates of $r^{2}$ between adjacent SNPs of 0.43 and 0.46, respectively [17], while Badke et al. [26] reported $r^{2}$ estimates of 0.46, 0.44, 0.36 and 0.39 for Duroc, Hampshire, Landrace and Yorkshire breeds, respectively. These values are comparable to the average $r^{2}$ values observed for LW (0.50) and LA (0.46) in our study. 
Table 2 Number of shared runs of homozygosity per chromosome between the Landrace and Large White populations

\begin{tabular}{lrc}
\hline Chromosome & Number of shared regions & $\begin{array}{l}\text { Number of SNPs } \\
\text { per chromosome }\end{array}$ \\
\hline SSC1 & 171 & 6378 \\
SSC2 & 94 & 2645 \\
SSC3 & 82 & 2201 \\
SSC4 & 77 & 3504 \\
SSC5 & 63 & 2168 \\
SSC6 & 51 & 2118 \\
SSC7 & 72 & 3347 \\
SSC8 & 65 & 2223 \\
SSC9 & 95 & 2615 \\
SSC10 & 52 & 1347 \\
SSC11 & 55 & 1769 \\
SSC12 & 29 & 1123 \\
SSC13 & 83 & 3562 \\
SSC14 & 89 & 3866 \\
SSC15 & 89 & 2609 \\
SSC16 & 50 & 1553 \\
SSC17 & 63 & 1609 \\
SSC18 & 53 & 1129 \\
Total & 1333 & 45,766 \\
\hline
\end{tabular}

Slightly larger LD estimates were observed at shorter distances for LW than for LA (Fig. 1), which suggests that the LW line was derived from a population that had a smaller ancient $N_{e}\left(N_{e 1000}=572\right)$ than the LA breed $\left(N_{e 1000}=740\right)$. Low $N_{e}$ in recent generations (Fig. 3) has led to the fixation of some LD blocks in LA and LW. Some of these LD blocks overlapped, as was observed in the shared $\mathrm{ROH}$ between the two breeds (Table 2). Average $r^{2}$ between adjacent SNPs that were calculated by using only one in every 10 available SNPs were equal to 0.27 and 0.30 for LA and LW, respectively. According to Meuwissen et al. [35], the average $r^{2}$ between adjacent SNPs needs to be greater than 0.2 for genomic selection (GS) to be effective. Thus, within the LA and LW populations evaluated here, $\mathrm{LD}$ is present at useful levels (i.e. $\left.r^{2}>0.3\right)[36,37]$ and SNP panels with sparser densities could be used for genome-wide association studies (GWAS) and GS. This is in agreement with Badke et al. [26] and Veroneze et al. [38], who reported that LD levels extend further in the European swine genome than in the bovine genome, which implies that less dense SNP panels can be used to conduct GWAS and GS in pigs. Low-density custom SNP panels could represent a cost-effective alternative for commercial breeding programs that aim at incorporating genomic tools in routine testing, while having little impact on the accuracy of GS.
Correlation of linkage phase $(r)$, which can be used to infer the history of a species and relatedness of breeds within species [12, 39], showed moderate values for LA and LW. For SNPs that were up to $50 \mathrm{~kb}$ apart, the average $r$ was equal to 0.77 . However, $r$ dropped sharply as the distance between SNPs increased, which indicates that estimation of SNP effects on performance traits of LA pigs based on SNP effects derived from LW pigs, and vice versa, could only be accurate if denser SNP panels were used, with distances between SNPs less than $5 \mathrm{~kb}$. Similar results were found for North American purebred pig populations, with pairwise $r$ at $10-50 \mathrm{~kb}$ between the Duroc, Hampshire, Landrace and Yorkshire populations ranging from 0.76 to 0.84 [26]. Our results indicate that the average gametic phase of two SNPs that are in LD is similar in both breeds analyzed. However, the phase correlations for pairs of SNPs that we observed between the LA and LW populations with the Illumina PorcineSNP60 Beadchip, limit the accuracy of inferring linkage phase in one breed based on estimates obtained from the other breed. To perform across-breed genomic prediction, $r$ values higher than 0.8 are needed for SNP effects to remain consistent across breeds $[14,39]$ and, thus, denser SNP panels are required for these populations. Hence, GS based on the Illumina PorcineSNP60 Beadchip must be performed within breed.

In pigs, selection is carried out on pure lines to improve performance of crossbred animals, which imposes a challenge to the implementation of GS since phenotypes of interest are measured on crossbred animals and for a small number of pure lines. Veroneze et al. [40] reported low levels of accuracy for GS when using across-population data in pigs, possibly due to the low relationship among breeds with different LD levels.

Chromosomal segments that contain homozygous SNP genotypes can be used to infer possible haplotypes that are inherited by the same individual, and subsequently estimate genomic inbreeding coefficients using $\mathrm{ROH}\left(F_{\mathrm{ROH}}\right)$ [41]. Furthermore, $\mathrm{ROH}$ length is negatively correlated with time of coancestry, since long $\mathrm{ROH}$ are observed when recent consanguinity events occur within a pedigree. In contrast, shorter $\mathrm{ROH}$ are produced by IBD genomic regions from old ancestors and are indicative of more ancient relatedness, which is frequently unaccounted for in an individual's recorded pedigree [12]. On average, longer stretches of $\mathrm{ROH}$ were observed for LW $(\sim 280 \mathrm{Mb})$ than for LA $(\sim 252 \mathrm{Mb})\left(\mathrm{p}<2.2^{\mathrm{e}-16}\right)$, which could be due to differences in the number of generations and selection strategy used by the company, with different levels of selection pressure on the traits to which these lines have been subjected to. These results are in agreement with the $N_{e}$ estimates that were obtained for the current generation of each breed, with the LA population having a larger $N_{e}$ than the LW population. 
Inbreeding levels for the base LA and LW populations based on the available pedigree are unknown, thus levels of inbreeding based on $F_{\mathrm{x}}$ were expected to be lower than levels of inbreeding based on $F_{\mathrm{ROH}}$ and $F_{\mathrm{SNP}}$. Correlations between $F_{\mathrm{x}}$ and $F_{\mathrm{ROH}}$ estimates were low for both the LA (0.24) and LW (0.015) breeds. These results are in agreement with previous reports on populations of Wagyu cattle [4], Iberian pigs [19], and Duroc, Large White and Pietrain commercial pig lines [42]. Pedigreebased estimates assume that there is no inbreeding in the base population and thus this contributes to the low correlation. In addition, the use of genomic information allows Mendelian sampling effects to be estimated more accurately and thus improves the estimates of inbreeding rate [43]. The SNP-by-SNP inbreeding coefficient measures the increase in frequency of homozygous genotypes, including both IBD and IBS alleles (Table 1). Thus, $F_{S \mathrm{NP}}$ can overestimate the levels of inbreeding compared to $F_{\mathrm{ROH}}$ and $F_{\mathrm{x}}$. The correlation between the genomic inbreeding estimates using $F_{\mathrm{ROH}}$ and $F_{\mathrm{SNP}}$ were high in our study $\left(r^{2}=0.7\right)$, which is in agreement with previous results $[18,19]$.

Some studies have reported weak correlations between inbreeding estimates obtained from pedigree and genotype data in several species, such as Wagyu cattle [4], Duroc, Large White and Pietrain commercial pig lines [42], sheep and birds [44]. Lopes et al. [42] identified a low correlation (0.27) between inbreeding estimates based on SNP data versus pedigree data for a LW population. This correlation was higher than that found in our study between $F_{\mathrm{x}}$ and $F_{\mathrm{ROH}}$ for the LW population $\left(r^{2}=0.015\right)$. However, Lopes et al. [42] used a different methodology based on kinship and a considerably smaller number of SNPs, i.e. 28,740 compared to 47,069 SNPs in our study. In addition to differences in quality control thresholds, the difference in these correlations could also be due to distinct genetic backgrounds in these two LW populations. However, other studies have also reported high correlations between pedigree and genomic inbreeding estimates $[6,18,19]$. The high correlation found in $[18$, 19] can be partially explained by the low $N_{e}$ of their populations $\left(N_{e}=10\right)$, which results in high levels of inbreeding (as in [18] i.e. $F_{\mathrm{x}}=0.35$ and $F_{\mathrm{SNP}}=0.8$ ). In this small Iberian pig population that has a low $N_{e}, F_{\mathrm{x}}$ was possibly sufficiently accurate, especially because complete pedigree information was available. In this situation, genomic information may not add much to the inbreeding estimates, which results in a high correlation between genomic and pedigree-based inbreeding estimates. In our study, $N_{e}$ in the last five generations varied between 40 and 53 in both populations and rigorous inbreeding control was applied. Therefore, low levels of inbreeding were observed and genomic inbreeding estimates were expected to be closer to the real inbreeding level than $F_{\mathrm{x}}$, which explains the low correlations found in our study. Saura et al. [19] reported a low negative correlation between $F_{\mathrm{x}}$ and $F_{\mathrm{ROH} \text { Short }}\left(r^{2}=-0.24\right)$ in populations with high levels of pedigree-based inbreeding $\left(F_{\mathrm{x}}=0.39\right)$ and SNP-by-SNP inbreeding $\left(F_{\mathrm{SNP}}=0.86\right)$. This negative correlation was explained by the fact that their $F_{\mathrm{ROH}}$ estimates were based only on short segments of $\mathrm{ROH}(0.5$ to $5 \mathrm{Mb}$ ), which mostly account for old inbreeding events. When long $\mathrm{ROH}$ segments $(>5 \mathrm{Mb})$ were included in the analysis, the correlation between $F_{\mathrm{x}}$ and $F_{\mathrm{ROH}}$ improved and reached a value of 0.63 . Our results showed that 76.1 and $76.8 \%$ of the $\mathrm{ROH}$ segments were short $(<5 \mathrm{Mb})$ for the LA and LW breeds, respectively, with an average length of $4.79 \mathrm{Mb}$ for LA and 4.55 Mb in LW. Our estimates of $F_{\mathrm{ROH}}$ included predominantly short fragments, as mentioned above, which could explain the low correlation between $F_{\mathrm{x}}$ and $F_{\mathrm{ROH}}$ that we observed.

Although we found low correlations between inbreeding estimates using genomic and pedigree data, we did observe higher levels of homozygosity in the LW than in the LA breeds with all three methods. Both lines have been selected for several years but the LW breed was imported eight years earlier than the LA breed. Since 2006, selection pressure has increased in both lines, and mating between close relatives is avoided to minimize the rate of inbreeding per generation. The number of animals maintained in the herd was larger for the LA breed than for the LW breed and selection intensity was stronger on the LA than on the LW population but lower levels of homozygosity were observed for the LA population. This could be due to the larger average $N_{e}$ maintained during the past generations for LA than for LW (Fig. 3) or to lower coancestry between the individuals selected as breeders.

Pedersen et al. [45] and Sonesson et al. [16] proposed the inclusion of genomic inbreeding information for GS, because estimation of inbreeding, based on pedigree information only, underestimates the levels of inbreeding and does not consider unaccounted parentage errors that can accumulate through generations. This is in agreement with our findings, which indicate the need to reconsider the weaknesses that are associated with estimates of population diversity that are based on pedigree information only.

\section{Conclusions}

The use of genomic data to evaluate population diversity in livestock animals can provide new and more precise insights into the effects of intense selection on production traits. Resulting information and knowledge can be used to effectively increase response to selection by appropriately managing the rate of inbreeding, 
minimizing negative effects from inbreeding depression, and maintaining desirable levels of genetic diversity. For populations with a low level of inbreeding, the use of genomic information has greatly increased the accuracy of genetic diversity estimates. Therefore, major shortand long-term positive impacts of selection response are expected as genomic data is widely incorporated into commercial or cooperative-based breeding programs of all sizes.

\section{Authors' contributions}

RZ contributed to data analysis and interpretation, and to writing and formatting of the manuscript. JOP and FFC contributed to the experimental design, data analysis and interpretation, and manuscript formatting and writing. LLC and PB contributed to data analysis and interpretation, and manuscript writing. AO and MSF contributed to the experimental design and sample acquisition. ARC contributed to the experimental design, data interpretation and manuscript writing. MCL contributed to the experimental design, data analysis and interpretation, manuscript writing, and supervised the work. All authors read and approved the final manuscript.

\section{Author details}

1 Embrapa Swine and Poultry National Research Center, Animal Breeding and Genetics, Concordia, SC, Brazil. ${ }^{2}$ Embrapa Southern Region Animal Husbandry, Bagé, RS, Brazil. ${ }^{3}$ BRF/SA, Curitiba, PR, Brazil. ${ }^{4}$ Programa de pós-graduação em Zootecnia/UFPel, Pelotas, RS, Brazil. ${ }^{5}$ Embrapa Recursos Genéticos e Biotecnologia, Brasília, DF, Brazil. ${ }^{6}$ Present Address: Faculdade de Agronomia e Medicina Veterinária (FAMV), University of Passo Fundo, Passo Fundo, RS, Brazil. ${ }^{7}$ Programa de pós-graduação em Ciências Animais/Universidade de Brasília, Brasília, DF, Brazil. ${ }^{8}$ Programa de pós-graduação em Zootecnia/Campus UDESC Oeste, Universidade do Estado de Santa Catarina, Chapecó, SC, Brazil.

\section{Acknowledgements}

The authors are grateful to AL. Tessmann for technical assistance. This work was funded by the Project 02.09.07.006.00.00 from the Brazilian Agricultural Research Corporation (EMBRAPA). R. Zanella was supported by a BJT Grant No. 373167/2012-1 from the National Council of Scientific and Technological Development (CNPq-Brazil). L.L. Cardoso is a PNPD/CAPES fellow. F.F. Cardoso and A.R. Caetano are CNPq fellows.

\section{Competing interests}

The authors declared that they have no competing interests.

Received: 20 January 2015 Accepted: 15 March 2016

Published online: 30 March 2016

\section{References}

1. ABCS 2013. http://www.abcs.org.br/images/pdf/registro.pdf. Accessed 14 Oct 2014.

2. Thornton PK. Livestock production: recent trends, future prospects. Philos Trans R Soc Lond B Biol Sci. 2010;365:2853-67.

3. Weigel KA. Controlling inbreeding in modern breeding programs. J Dairy Sci. 2001;84:E177-84.

4. Scraggs E, Zanella R, Wojtowicz A, Taylor JF, Gaskins CT, Reeves JJ, et al. Estimation of inbreeding and effective population size of full-blood wagyu cattle registered with the American Wagyu Cattle Association. J Anim Breed Genet. 2014;131:3-10.

5. Burrow HM. The effects of inbreeding in beef cattle. Anim Breed Abstr. 1993;61:737-51.

6. Silió L, Rodríguez MC, Fernández A, Barragán C, Benítez R, Óvilo C, et al. Measuring inbreeding and inbreeding depression on pig growth from pedigree or SNP derived metrics. J Anim Breed Genet. 2013;130:349-60.

7. Zanella R, Settles ML, McKay SD, Schnabel R, Taylor J, Whitlock RH, Schukken Y, et al. Identification of loci associated with tolerance to Johne's disease in Holstein cattle. Anim Genet. 2011;42:28-38.
8. Wilson K, Zanella R, Ventura C, Johansen HL, Framstad T, Janczak A, et al. Identification of chromosomal locations associated with tail biting and being a victim of tail-biting behavior in the domestic pig (Sus scrofa domesticus). J Appl Genet. 2012;53:449-56.

9. Sanchez MP, Tribout T, lannuccelli N, Bouffaud M, Servin B, Tenghe A, et al. A genome wide association study of production traits in a commercial population of Large White pigs: evidence of haplotypes affecting meat quality. Genet Sel Evol. 2014;46:12.

10. Ramos AM, Crooijmans RPMA, Affara NA, Amaral AJ, Archibald AL, Beever $J$ E, et al. Design of a high density SNP genotyping assay in the pig using SNPs identified and characterized by next generation sequencing technology. PLoS One. 2009;4:e6524.

11. Bosse M, Megens HJ, Madsen O, Paudel Y, Frantz LAF, Schook LB, et al. Regions of homozygosity in the porcine genome: consequence of demography and the recombination landscape. PLoS Genet. 2012;8:e1003100.

12. Kirin M, McQuillan R, Franklin CS, Campbell H, Mckeigue PM, Wilson JF. Genomic runs of homozygosity record population history and consanguinity. PLoS One. 2010;5:e13996.

13. Meuwissen TH, Goddard ME. Prediction of identity by descent probabilities from marker-haplotypes. Genet Sel Evol. 2001;33:605-34.

14. de Roos APW, Schrooten C, Veerkamp RF, van Arendonk JAM. Effects of genomic selection on genetic improvement, inbreeding, and merit of young versus proven bulls. J Dairy Sci. 2011;94:1559-67.

15. Uimari P, Tapio M. Extent of linkage disequilibrium and effective population size in Finnish Landrace and Finnish Yorkshire pig breeds. J Anim Sci. 2011;89:609-14.

16. Sonesson AK, Woolliams JA, Meuwissen TH. Genomic selection requires genomic control of inbreeding. Genet Sel Evol. 2012;44:27.

17. Wang L, Sørensen P, Janss L, Ostersen T, Edwards D. Genome-wide and local pattern of linkage disequilibrium and persistence of phase for 3 Danish pig breeds. BMC Genet. 2013;14:115.

18. Saura M, Fernández A, Rodríguez MC, Toro MA, Barragán C, Fernández Al et al. Genome-wide estimates of coancestry and inbreeding in a closed herd of Iberian pigs. PLoS One. 2013;8:e78314.

19. Saura M, Fernández A, Varona L, Fernández Al, de Cara MAR, Barragán C, et al. Detecting inbreeding depression for reproductive traits in Iberian pigs using genome-wide data. Genet Sel Evol. 2015;47:1.

20. Gibson J, Morton NE, Collins A. Extended tracts of homozygosity in outbred human populations. Hum Mol Genet. 2006;15:789-95.

21. Zhu M, Zhu B, Wang YH, Wu Y, Xu L, Guo LP, et al. Linkage disequilibrium estimation of Chinese beef Simmental cattle using high-density SNP panel. Asian-Australas J Anim Sci. 2013;26:772-9.

22. Purcell S, Neale B, Todd-Brown K, Thomas L, Ferreira MAR, Bender D, et al PLINK: a toolset for whole-genome association and population based linkage analysis. Am J Hum Genet. 2007;81:559-75.

23. Clayton D. snpStats: SnpMatrix and XSnpMatrix classes and methods. R package version 1.14.0; 2013.

24. Sargolzaei M, Chesnais JP, Schenkel FS. A new approach for efficient genotype imputation using information from relatives. BMC Genomics. 2014;15:478.

25. Weir BS. Genetic data analysis II: methods for discrete population genetic data. 2nd ed. Sunderland: Sinauer Associates; 1996.

26. Badke YM, Bates RO, Ernst CW, Schwab C, Steibel JP. Estimation of linkage disequilibrium in four US pig breeds. BMC Genomics. 2012;13:24.

27. Sved JA. Linkage disequilibrium and homozygosity of chromosome segments in finite populations. Theor Popul Biol. 1971;2:125-41.

28. Hayes BJ, Visscher PM, MCPartlan HC, Goddard ME. Novel multilocus measure of linkage disequilibrium to estimate past effective population size. Genome Res. 2003;13:635-43.

29. Corbin LJ, Blott SC, Swinburne JE, Vaudin M, Bishop SC, Woolliams JA. Linkage disequilibrium and historical effective population size in the Thoroughbred horse. Anim Genet. 2010;41:8-15.

30. Wright S. Evolution in Mendelian genetics. Genetics. 1931;16:97-159.

31. Nomura T, Honda T, Mukai F. Inbreeding and effective population size of Japanese Black cattle. J Anim Sci. 2001;79:366-70.

32. Kent WJ, Sugnet CW, Furey TS, Roskin KM, Pringle TH, Zahler AM, et al. The human genome browser at UCSC. Genome Res. 2002;12:996-1006.

33. Wang SJ, Liu WJ, Sargent CA, Zhao SH, Liu HB, Liu XD, et al. Effects of the polymorphisms of $M \times 1, B A T 2$ and $C X C L 12$ genes on immunological traits in pigs. Mol Biol Rep. 2012;39:2417-27. 
34. Antelman J, Manandhar G, Yi YJ, Li R, Whitworth KM, Sutovsky M, Agca C, et al. Expression of mitochondrial transcription factor A (TFAM) during porcine gametogenesis and preimplantation embryo development. J Cell Physiol. 2008;217:529-43.

35. Meuwissen TH, Hayes BJ, Goddard ME. Prediction of total genetic value using genome-wide dense marker maps. Genetics. 2001;157:1819-29.

36. Ardlie KG, Kruglyak L, Seielstad M. Patterns of linkage disequilibrium in the human genome. Nat Rev Genet. 2002;3:299-309.

37. Sargolzaei M, Schenkel FS, Jansen GB, Schaeffer LR. Extent of linkage disequilibrium in Holstein cattle in North America. J Dairy Sci. 2008;91:2106-17.

38. Veroneze R, Lopes PS, Guimarães SEF, Silva FF, Lopes MS, Harlizius B, et al Linkage disequilibrium and haplotype block structure in six commercial pig lines. J Anim Sci. 2013;91:3493-501.

39. de Roos APW, Hayes BJ, Spelman RJ, Goddard ME. Linkage disequilibrium and persistence of phase in Holtein-Friesian, Jersey and Angus cattle. Genetics. 2008;79:1503-12.
40. Veroneze R, Lopes MS, Hidalgo AM, Guimarães SE, Silva FF, et al. Accuracy of genome-enabled prediction exploring purebred and crossbred pig populations. J Anim Sci. 2015;93:4684-91.

41. Bjelland DW, Weigel KA, Vukasinovic N, Nkrumah JD. Evaluation of inbreeding depression in Holstein cattle using whole-genome SNP markers and alternative measures of genomic inbreeding. J Dairy Sci. 2013;96:4697-706.

42. Lopes MS, Silva FF, Harlizius B, Duijvesteijn N, Lopes PS, Guimarães SEF, et al. Improved estimation of inbreeding and kinship in pigs using optimized SNP panels. BMC Genet. 2013;14:92.

43. Daetwyler HD, Villanueva B, Bijma P, Woolliams JA. Inbreeding in genomewide selection. J Anim Breed Genet. 2007;124:369-76.

44. Pemberton J. Measuring inbreeding depression in the wild: the old ways are the best. Trends Ecol Evol. 2010;19:613-5.

45. Pedersen LD, Sørensen AC, Berg P. Marker-assisted selection reduces expected inbreeding but can result in large effects of hitchhiking. J Anim Breed Genet. 2010;127:189-98.

\section{Submit your next manuscript to BioMed Central and we will help you at every step:}

- We accept pre-submission inquiries

- Our selector tool helps you to find the most relevant journal

- We provide round the clock customer support

- Convenient online submission

- Thorough peer review

- Inclusion in PubMed and all major indexing services

- Maximum visibility for your research

Submit your manuscript at www.biomedcentral.com/submit
Biomed Central 